\title{
Recent advances in ultrasonic-assisted machining for the fabrication of micro/nano-textured surfaces
}

(C) The Author(s) 2017. This article is published with open access at link.springer.com and journal.hep.com.cn

\begin{abstract}
In this paper, the state of art of ultrasonicassisted machining technologies used for fabrication of micro/nano-textured surfaces is reviewed. Diamond machining is the most widely used method in industry for manufacturing precision parts. For fabrication of fine structures on surfaces, conventional diamond machining methods are competitive by considering the precision of structures, but have limitations at machinable structures and machining efficiency, which have been proved to be partly solved by the integration of ultrasonic vibration motion. In this paper, existing ultrasonic-assisted machining methods for fabricating fine surface structures are reviewed and classified, and a rotary ultrasonic texturing (RUT) technology is mainly introduced by presenting the construction of vibration spindles, the texturing principles, and the applications of textured surfaces. Some new ideas and experimental results are presented. Finally, the challenges in using the RUT method to fabricate micro/ nano-textured surfaces are discussed with respect to texturing strategies, machinable structures, and tool wear.
\end{abstract}

Keywords ultrasonic-assisted machining, textured surface, micro/nano-structures, functional performance

\section{Introduction}

The scientific importance and industrial value of functional micro/nano-textured surfaces are getting increasing attention. Typical examples include optical retroreflective and

Received October 14, 2016; accepted December 4, 2016

Shaolin XU, Tsunemoto KURIYAGAWA ( $₫)$

Division of Biomechanical Engineering, Graduate School of Biomedical Engineering, Tohoku University, Sendai 980-8579, Japan

E-mail: tkuri@cc.mech.tohoku.ac.jp

Keita SHIMADA, Masayoshi MIZUTANI

Department of Mechanical Systems Engineering, Graduate School of Engineering, Tohoku University, Sendai 980-8579, Japan antireflective structures [1], medical biocompatible [2] and antimicrobial structures [3], and tribological friction tunable structures [4], which have been widely studied and industrially applied. To efficiently fabricate tailored structures on surfaces to get these functional performances is of great industrial value. Recently, the rapid development of biomimetics has also given much inspiration to researchers when designing functional textured surfaces [5]. However, the reproduction or imitation of structures on surfaces of organisms in nature has been found to be a great challenge for engineers, because many useful nature surfaces possess complex hybrid, multi-layer or directional structures with a scale as small as nanometers. Thus, utilizing the existing methods for such purpose would be unfeasible or time-consuming. To satisfy the requirements, researchers are always developing new methods or improving the capability of existing methods. Many technologies have been established for the fabrication of functional textured surfaces [6].

Diamond machining as the most widely used method for manufacturing precision parts is also capable of fabricating various surface structures, which have been illustrated in a published review paper [7]. It can be found that the machinable structures are limited and the machining efficiency is dependent on machined structures. Our group has also been focusing on researching and exploring new diamond machining methods for the efficient fabrication of micro/nano-textured surfaces with high precision. Ultrasonic-assisted machining as a traditional method to machine hard-to-machine materials has gotten our attention. The high-frequency tool-work interaction induced by ultrasonic vibration has been proved to be useful in the fabrication of micro/nano-textured surfaces [8-12]. It can be noticed that some other researchers worldwide have also followed a similar principle in fabricating fine surface structures [13-16].

This paper briefly reviews the methods with the assist of ultrasonic vibration for fabrication of micro/nano-textured surfaces in Section 2, and then mainly addresses a new rotary ultrasonic texturing (RUT) method developed by 
our group in Sections 3 and 4. The texturing principles, machinable structures, and the merits and demerits of these ultrasonic-assisted machining methods are discussed as well. The entire development processes of the RUT method, including the development of applicable ultrasonic spindles, the calculation of surface generation processes, the designing of diamond tools, and the texturing strategies are focused. This new method provides designers with additional freedom to efficiently fabricate various fine structures on surfaces of different materials.

\section{Classification of ultrasonic-assisted texturing methods}

Ultrasonic-assisted machining refers to mechanical processing methods that apply high-frequency vibration (generally greater than $20 \mathrm{kHz}$ ) to a tool or a workpiece with its vibration amplitude ranging from several micrometers to several tens of micrometers [17]. There has been a large amount of research in terms of integrating ultrasonic vibration into conventional mechanical machining processes to achieve machining performances. Only those works aimed at fabricating surface structures will be discussed in this paper. We called ultrasonic-assisted machining for the fabrication of micro/nano-textured surface as ultrasonic-assisted texturing methods, which are classified into two categories according to the role of ultrasonic vibration motion in the present paper.

2.1 Ultrasonic-assisted machining for the fabrication of structures

In the first category, the surface fabrication mechanism is the same as that of conventional diamond machining methods, i.e., removing materials with cutting edges at a high speed to generate structures. The details of the mechanical fabrication processes for surface structures can be found in Ref. [7]. Figure 1 shows the various machinable structures. By integrating ultrasonic vibration motion to the conventional diamond machining methods, material removal efficiency can be improved and tool wear can be minimized, which is the role of ultrasonic vibration motion. Four typical ultrasonic-assisted diamond machining methods integrated with grinding, milling, cutting, and turning, are schematically shown in Fig. 2. There are usually two types of ultrasonic vibration modes, 1D (reciprocating) vibration mode and 2D (elliptical or circular) vibration mode. The integration of ultrasonic vibration to these conventional diamond machining processes can promote the successful fabrication of very fine structures as small as several micrometers or even nanometers dimensions, which is an impossible task without the assistance of ultrasonic vibration. For example, the most widely used elliptical vibration-assisted cutting technology has been used to successfully fabricate different micro/nanostructures on hardened steel [18] and various 3D microstructures on hard plated copper [19]. Figure 3 shows two typical surface structures. Another example, micro-grooves in a hard-to-machine material were successfully fabricated by ultrasonic-assisted grinding using a diamond grinding pin with a diameter of several tens of micrometers [20].

\subsection{Newly proposed ultrasonic-assisted texturing methods}

The second category consists of texturing methods that actively modulate the ultrasonic vibration motion. The surface structures are generated by controlling every step of the cutting motion at each vibration circle. These methods can be subdivided into several categories according to the type of integrated conventional diamond machining methods. Turning [13-15], grooving [21], and rotary machining [10-12] have been used to fabricate micro/nano-textured surfaces by integrating ultrasonic vibration motion. The cutting motion is achieved with a rotating cylindrical workpiece, a linearly-feed cutting tool, and a rotating cutting tool, respectively. The machinable

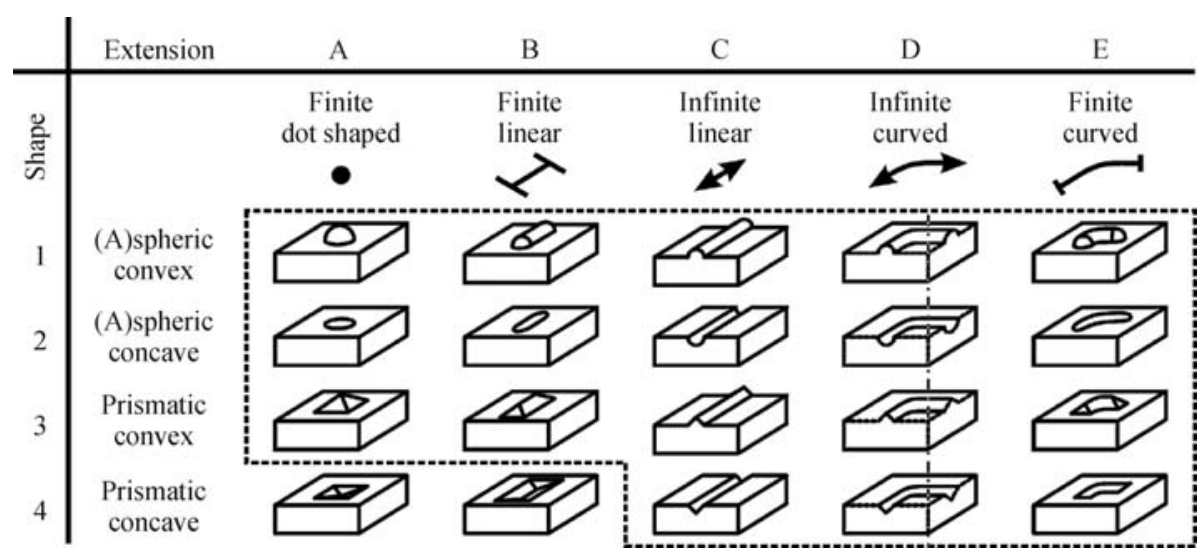

Fig. 1 Classification of machinable structures of diamond machining methods by shape and extension. Reprinted from Ref. [7] with permission from Elsevier 
(a)

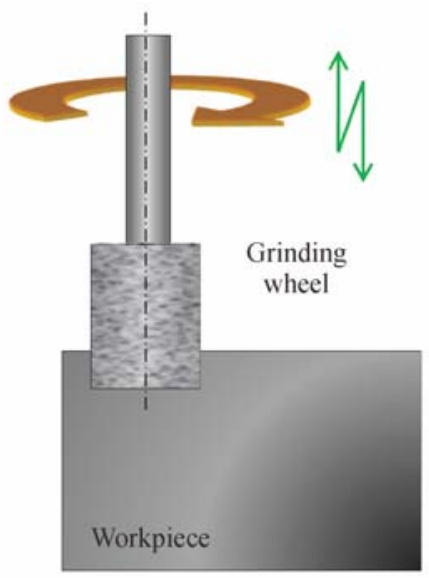

(c)

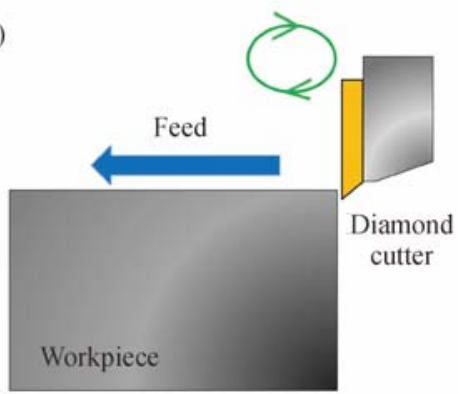

(b)

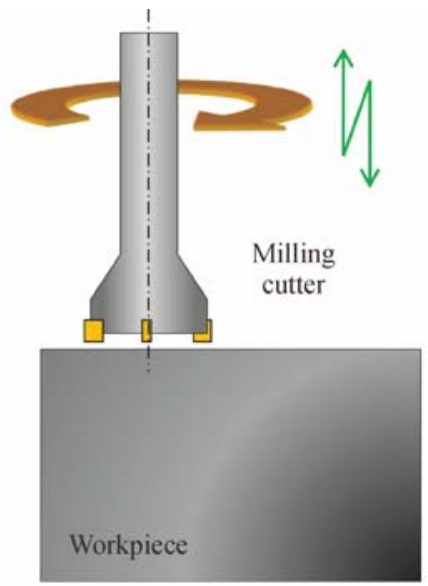

(d)

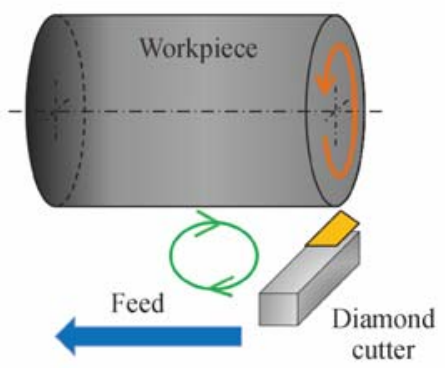

Fig. 2 Schematics of four types of typical ultrasonic-assisted machining processes. (a) 1D ultrasonic-assisted grinding; (b) 1D ultrasonic-assisted milling; (c) 2D ultrasonic-assisted cutting; (d) 2D ultrasonic-assisted turning
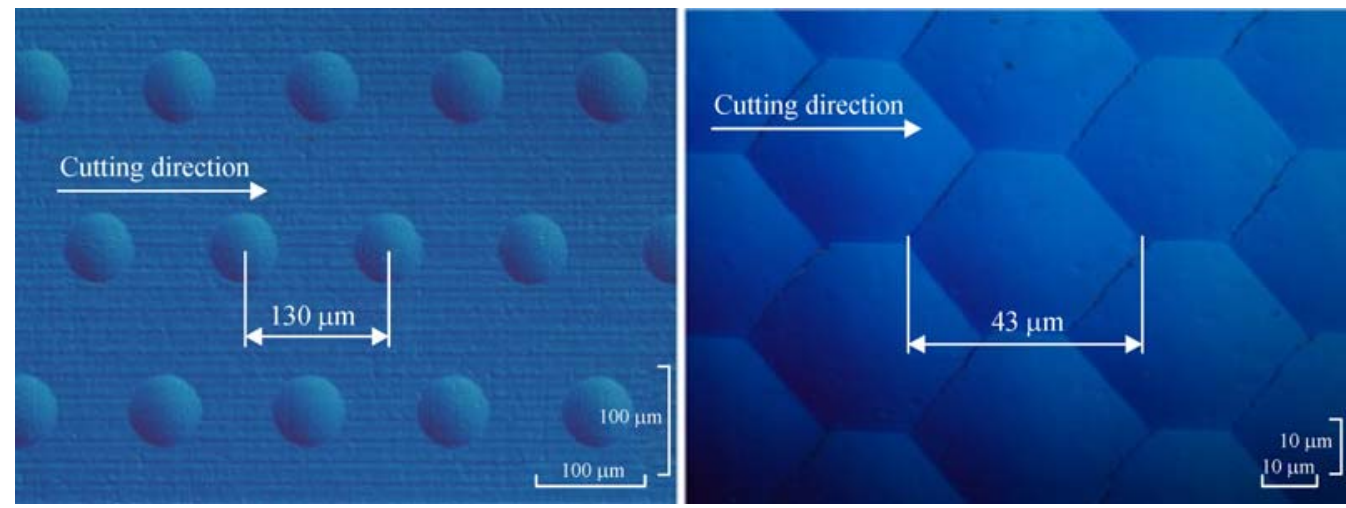

Fig. 3 Two typical structures fabricated with elliptical ultrasonic-assisted cutting technology. Reprinted from Ref. [18] with permission from Elsevier

structures mainly depend on tool geometry, vibration mode, feed path, and their combinations. Figure 4 illustrates the principle of ultrasonic-assisted turning processes for micro-texturing [13,14]. 1D and 2D vibration modes have been verified. When $1 \mathrm{D}$ vibration mode is applied, the continuous cutting process is transformed into an intermittent cutting process, allowing for the fabrication of microdimples. As for the 2D vibration mode, the authors developed a new elliptical ultrasonic vibration spindle that can make the tool vibrate in the cutting and depth-of-cut directions. By modulating the cutting depth and the vibration amplitude, a high-frequency intermittent contact between the cutting edge and workpiece was obtained, and intentionally controlled for the fabrication of surface meso/ micro-textures. Figure 5 shows two typical textured surface fabricated with this elliptical vibration assisted texturing method.

In our group, a novel RUT method was developed by integrating ultrasonic vibration into rotary machining processes. The combination of ultrasonic vibration, 


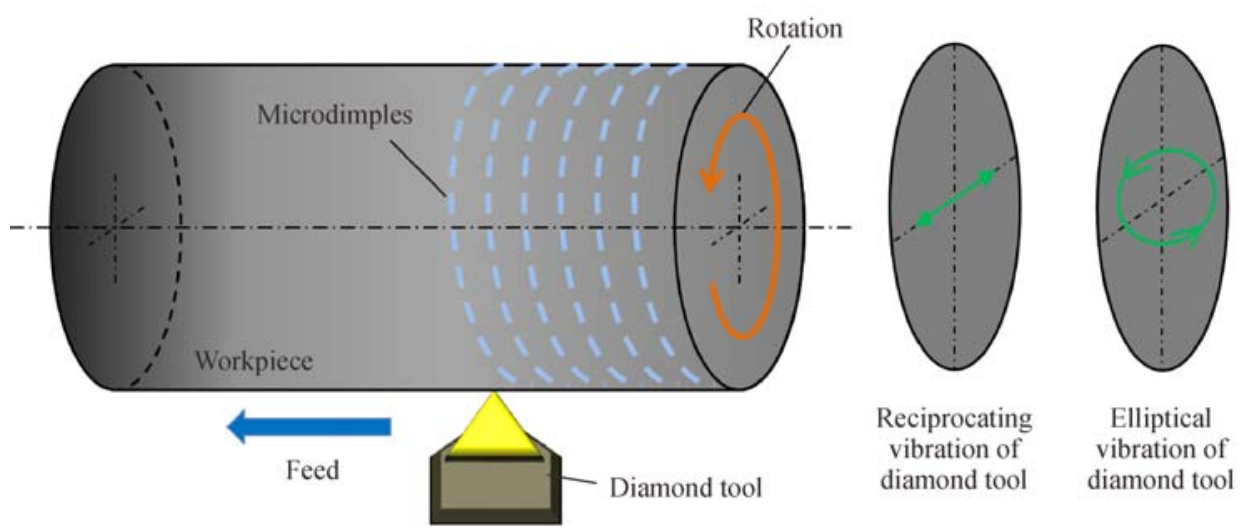

Fig. 4 1D and 2D ultrasonic-assisted turning processes for micro-texturing
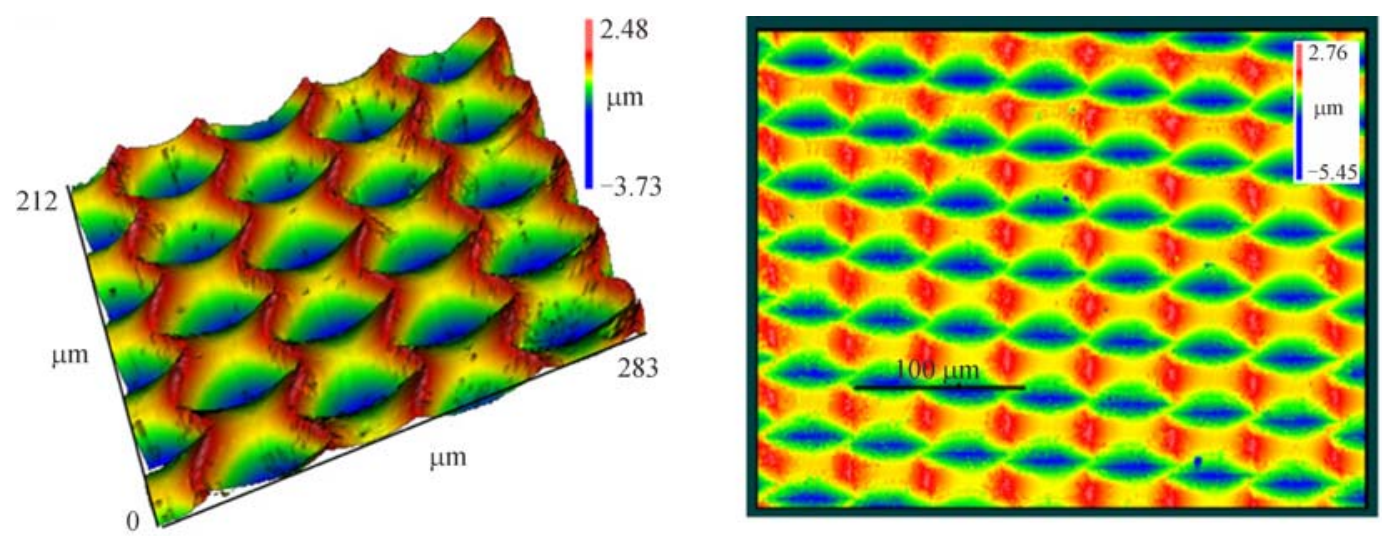

Fig. 5 Two typical structures fabricated with an elliptical ultrasonic texturing method with tools of different geometries. Reprinted from Ref. [15] with permission from Elsevier

rotation, and feed motion can lead to high-frequency periodic change in the cutting motion, which has been proposed to be deliberately controlled to fabricate micro/ nano-textured surfaces. A rotary ultrasonic spindle is theoretically applicable for fabricating structures on surface of any shape, making it more feasible than other ultrasonic spindles. However, the conventional rotary ultrasonic spindle can only generate $1 \mathrm{D}$ reciprocating vibration along the axis of the spindle, thereby limiting the applicable shapes of the cutting loci in the RUT process. If the diamond tool can freely vibrate in $3 \mathrm{D}$ space, a cutting locus with a considerably greater flexibility can be obtained, and more textural patterns can be fabricated.

Therefore, the structure of ultrasonic spindle plays a key role in the feasibility of the RUT method. A new 3D rotary ultrasonic spindle was proposed and designed by our group. Figure 6 shows the schematic of the proposed 3D RUT processes. A 3D rotary ultrasonic spindle can generate ultrasonic vibration along all three axes, namely, the longitudinal vibration (LV) along the axis of the spindle, the circular vibration $(\mathrm{CV})$ in the plane perpendicular to the axis of the spindle, and the hybrid vibration
(HV) in 3D space. The cutting locus of the cutting edge is deliberately modulated by controlling the resultant motion of the tool rotation, feed motion and vibration for fabricating surface textures. If the texturing parameters are appropriately controlled, micro/nanostructures are expected to be fabricated as schematically shown in Fig. 7. By controlling the feed motion, hybrid textured surfaces with primary structures along the feed direction and micro/nanostructures as secondary structures can be fabricated. The 3D RUT technique can be potentially used to fabricate different types of precisely controlled textural patterns at a high speed because of the high frequency of vibration motion and mechanical material removal mechanism.

\section{Rotary ultrasonic texturing}

\subsection{Construction of rotary ultrasonic spindles}

The vibration mode depends on the construction of the ultrasonic vibrator. In our study, a resonant piezoelectric 

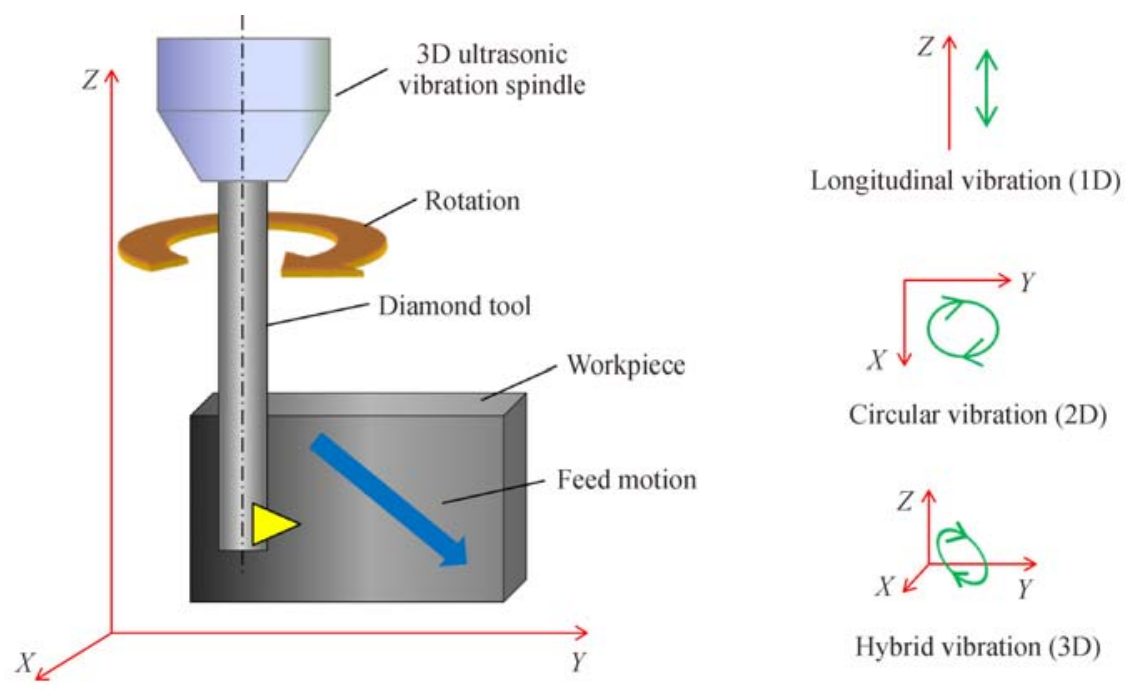

Circular vibration (2D)

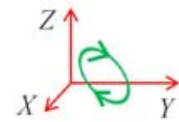

Hybrid vibration (3D)

Fig. 6 Concept of 3D RUT method using a 3D rotary ultrasonic spindle
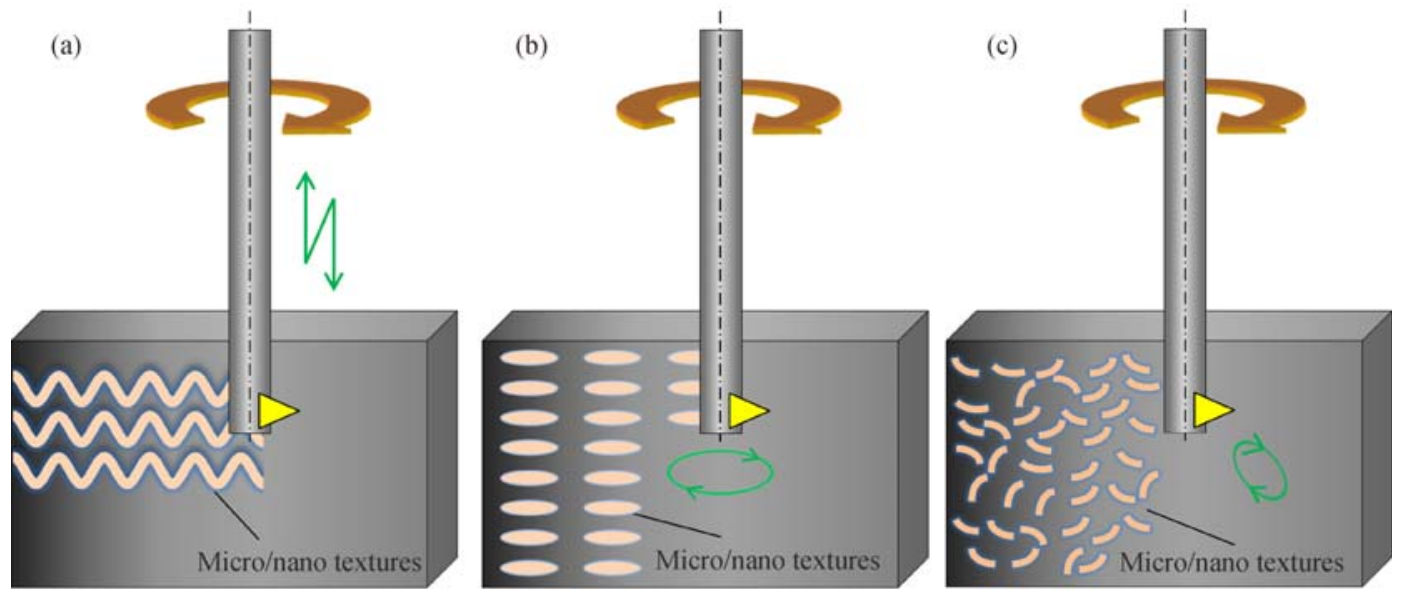

Fig. 7 Possible machinable structures of the RUT method under vibration modes of (a) LV, (b) CV, and (c) HV

vibrator was selected for manufacturing the rotary ultrasonic spindle. The vibrator was resonated by exciting several combined piezoelectric plates with high-frequency electrical signals, which are sandwiched with metal cylindrical horns. The high-frequency electrical energy was converted into mechanical vibration via the resonant piezoelectric transducer (PZT). The horn/tool assembly was used to amplify the vibration amplitude of the tool, because the oscillation amplitude at the face of the PZT was insufficient to achieve a reasonable cutting rate. Figure 8 shows two types of PZT systems for generating two basic ultrasonic vibration modes, namely, LV mode and bending vibration (BV) mode. The LV mode indicates that the tool vibrates along the axial $(Z)$ direction, and the BV mode indicates that the tool vibrates in the transverse $(X Y)$ plane perpendicular to the axis. To generate LV mode, the PZT utilizes only one set of round piezoelectric plates as shown in Fig. 8(a). When sinusoidal voltage is applied to the transducer, the piezoelectric plate expands and contracts, so that the vibrator is resonated and the tool tip attached to the end of the horn vibrates in the LV mode along the $Z$ axis. The vibration amplitude depends on the applied voltage, the material property of PZT and the spindle structures. The vibration amplitude is magnified by the horns, and maximized at the tool tip. The 1D ultrasonic vibration spindle has been widely used in the rotary ultrasonic machining processes. In Fig. 8(b), if two halfround piezoelectric plates are placed on the PZT and two sinusoidal voltages with $180^{\circ}$ phase difference are applied to the piezoelectric plates, the two piezoelectric plates will expand and contract alternately, ultimately causing the tool attached to the end of the horn to vibrate with the bending mode in the $X Y$ plane.

Different types of ultrasonic vibrators can be developed 
(a)

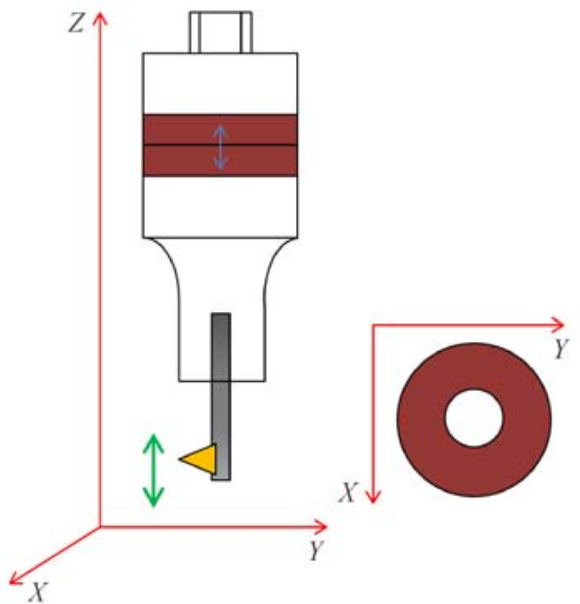

(b)

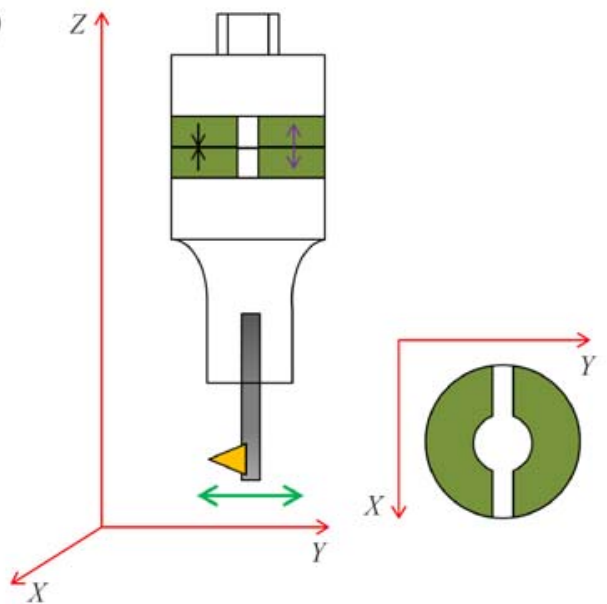

Fig. 8 Two types of PZT systems for generating the two basic ultrasonic vibration modes: (a) LV mode, (b) BV mode

by combining the two basic PZT systems. Figure 9 shows an ultrasonic vibrator that can generate $2 \mathrm{D}$ vibration in the transverse $(X Y)$ plane. Four piezoelectric plates with the same resonant frequency are placed on the ultrasonic actuator. Thus, the specific shape of the vibration locus depends on the vibration amplitudes and on the phase difference of the applied sinusoidal voltages. If sinusoidal voltages with $180^{\circ}$ phase difference are applied on every two opposite piezoelectric plates, two BV modes can be generated simultaneously. For a specific phase difference of the two BVs, an elliptical or circular vibration mode can be generated in the transverse $(X Y)$ plane. For example, the $\mathrm{CV}$ mode can be generated by applying $0^{\circ}, 90^{\circ}, 180^{\circ}$, and $270^{\circ}$ phase shifted sinusoidal signals having the same amplitude on the four piezoelectric plates in the clockwise direction.

In this paper, the PZT systems shown in Figs. 8(a) and 9
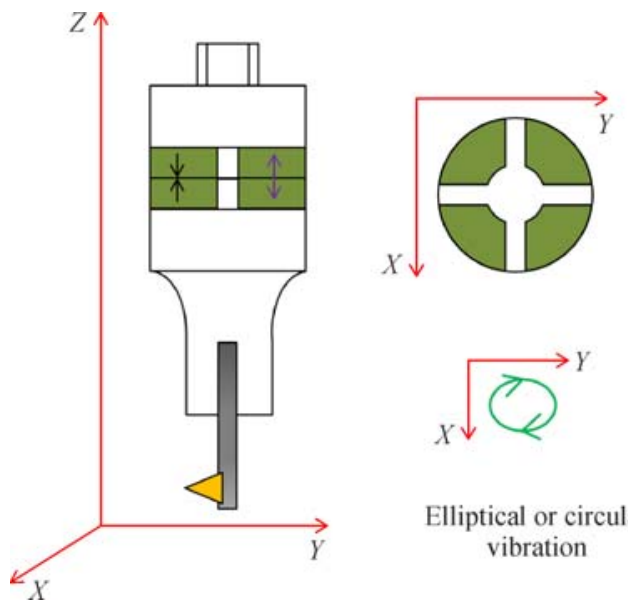

Elliptical or circular vibration

Fig. 9 PZT system for generating elliptical or circular vibration in the transverse $X Y$ plane were further combined into one ultrasonic vibrator shown in Fig. 10, and a new 3D hybrid ultrasonic vibrator was designed and manufactured. A circular vibration was tuned by modulating the abovementioned parameters for this vibrator. As such, the $3 \mathrm{D}$ ultrasonic vibrator can generate the LV mode along the axis ( $Z$ direction) of the spindle, the $\mathrm{CV}$ mode on the transverse $(X Y)$ plane, and the 3D HV in the 3D space by simultaneously implementing the LV and $\mathrm{CV}$ modes. Figure 11 schematically shows the construction of the ultrasonic vibration spindle. The main spindle is rotationally driven by a motor. The resonant ultrasonic vibrator controlled by an ultrasonic oscillator is attached coaxially to the spindle. Step horns are connected to the ultrasonic vibrator integrally. A tool, such as a grinding wheel or a cutting tool, is then mounted at the tip end of the horn. We used this spindle in conducting RUT experiments to fabricate micro/nano-textured surfaces.

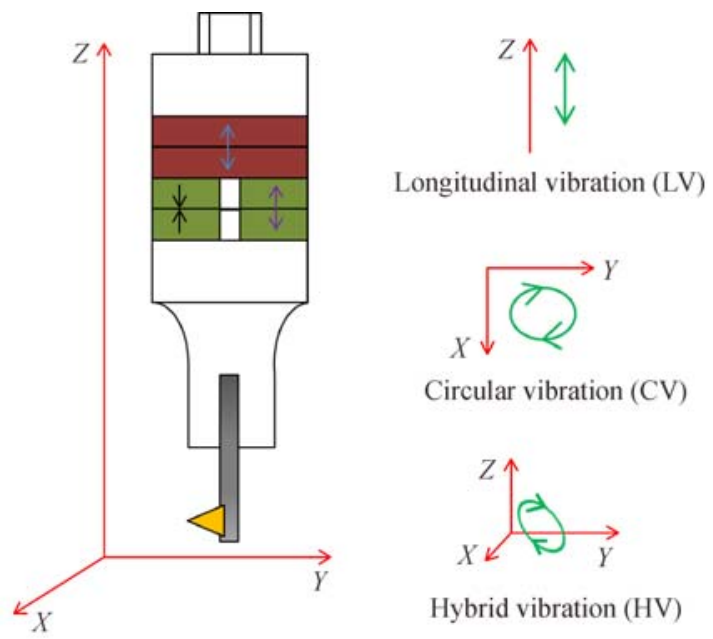

Fig. 10 PZT system used for manufacturing the 3D rotary ultrasonic spindle 


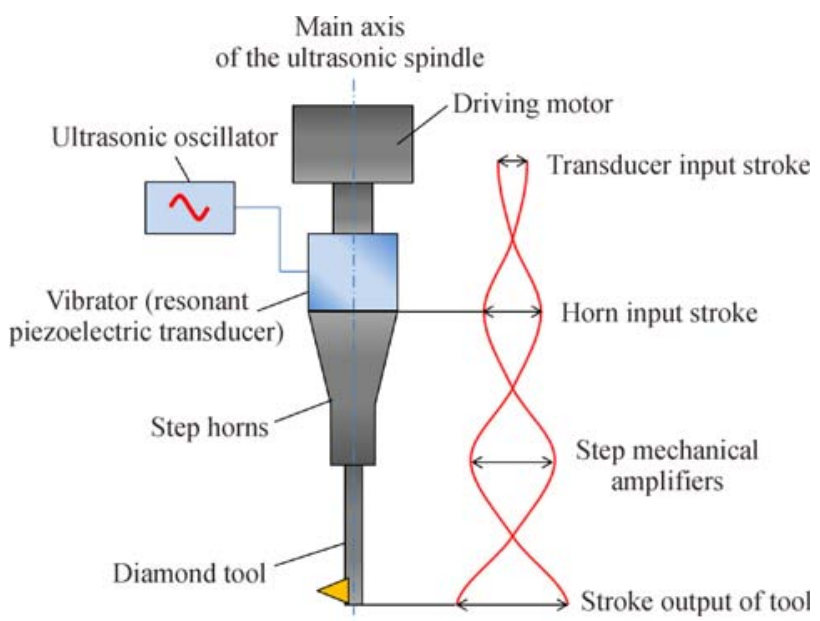

Fig. 11 Schematic of the construction of the 3D rotary ultrasonic spindle

(a)

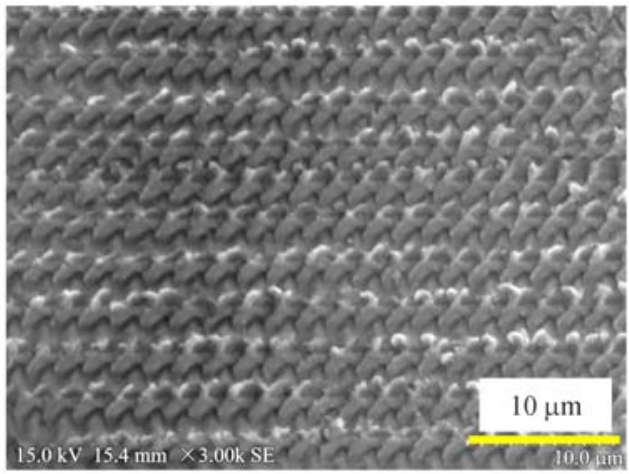

(b)

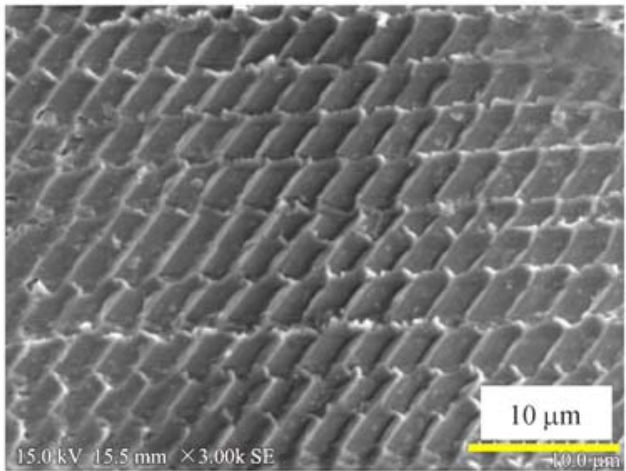

(c)

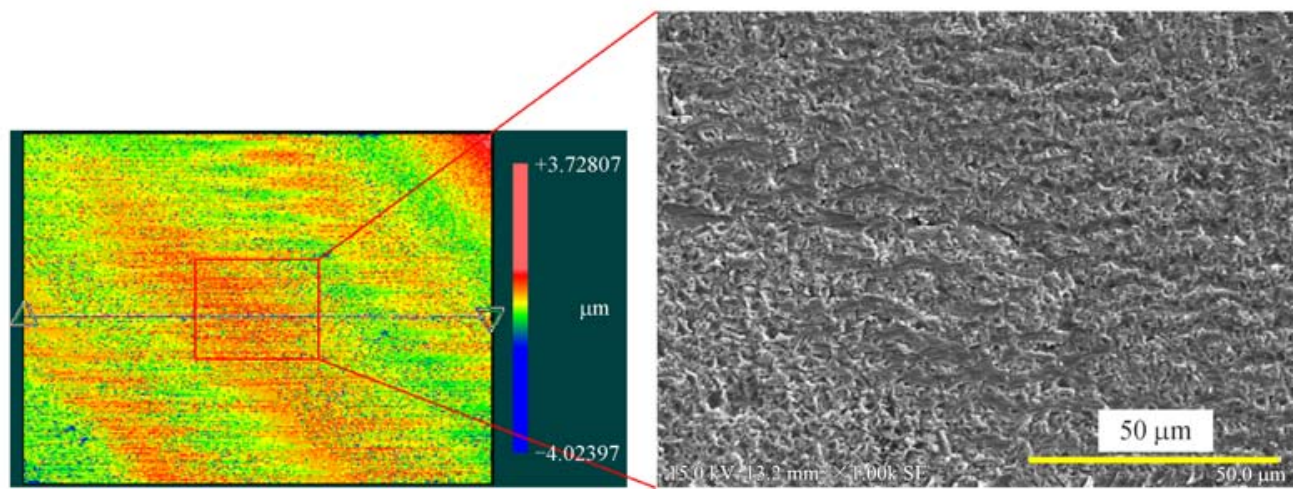

3.2 Texturing with diamond grinding wheels

The RUT method was inspired by an ultrasonic-assisted grinding method [22]. Periodic micro/nano-structures were observed on machined surfaces after ultrasonic-assisted slant-feed grinding (UASG) was performed. The details of its texturing mechanisms can be found in our previous work [8]. Diamond abrasives of irregular shapes on grinding wheels are generally randomly distributed. Therefore, surface structures with various patterns were observed on the machined surface. Figure 12 shows typical textured surfaces after UASG under three types of vibration modes. As shown, sinusoidal structures or structures along the sinusoidal locus were fabricated under LV mode; periodical micro-dimples or other micro-concave structures were fabricated under $\mathrm{CV}$ mode; and a random-like rough surface was successfully
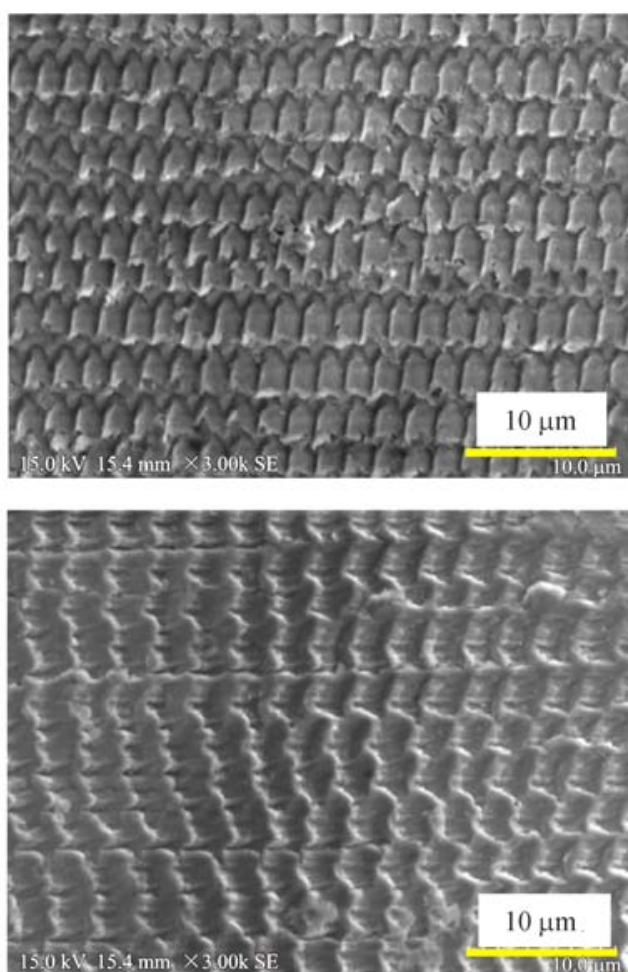

Fig. 12 Typical textured surfaces fabricated by UASG respectively under (a) LV, (b) CV, and (c) HV modes 
fabricated under 3D HV mode. These results proved that the RUT method can fabricate various structures at micrometer or even nanometer dimensions, provided that the diamond abrasives have appropriate geometries.

\subsection{Texturing with designed single point diamond tools}

Different types of textural patterns were obtained because of the irregular geometrical shapes of the diamond abrasives on the grinding wheels. The calculated cutting loci under three types of vibration modes shown in Fig. 13 can be used to gain insight into the fabrication of periodic textural patterns or random-like rough surfaces. However, no clear conclusion can be made as to what diamond abrasive can fabricate a specific textural pattern because of completely random distribution of the diamond abrasives on the grinding wheels. To make the texturing process

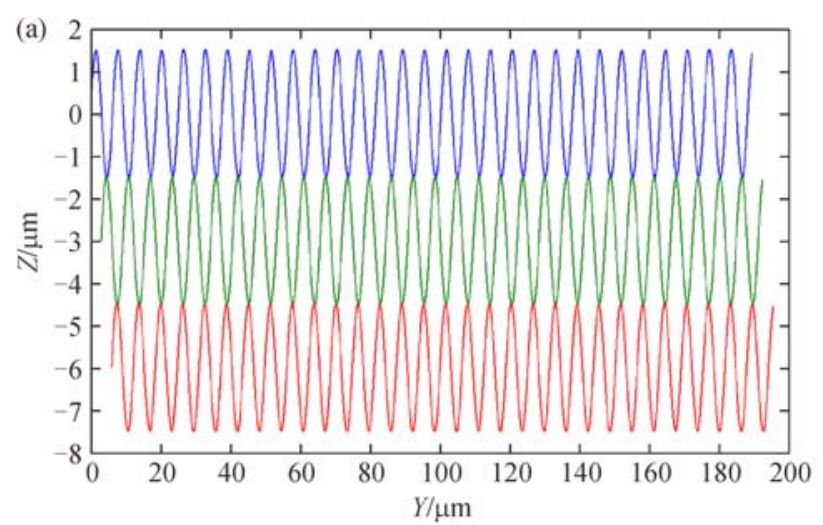

(b)

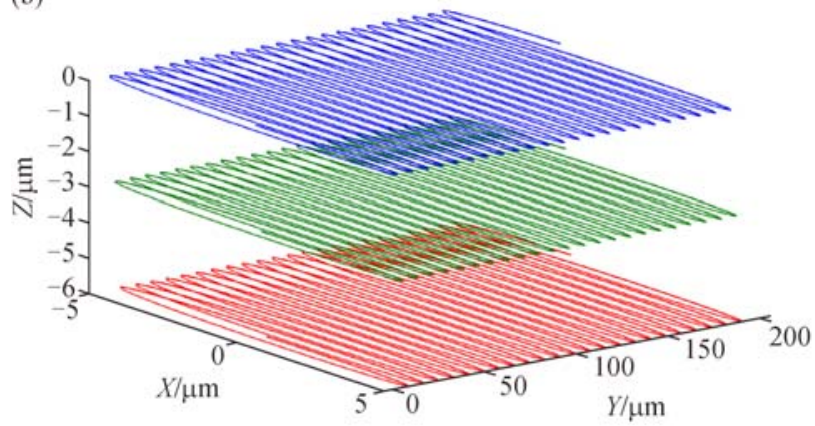

(c)

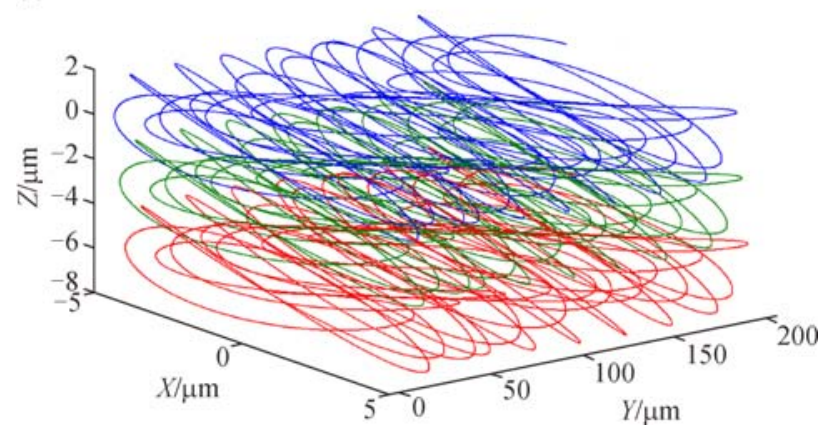

Fig. 13 Three typical cutting loci of the RUT processes. (a) LV, (b) $\mathrm{CV}$, and (c) HV vibration modes more controllable, the diamond cutting edges should be regularly distributed with fixed locations on the tool. Material removal mechanisms should be investigated with regard to designing texturing procedures, as well as the tools that can implement the RUT processes under different vibration modes.

Therefore, diamond tools with only one cutting tip, which is referred to as single-point diamond tools, were designed and manufactured. First, electroplated single point diamond tools were manufactured to examine material removal mechanisms. Specific RUT procedures under the LV and CV modes were developed. The cutting loci were mathematically calculated and drawn to predict the textural features that are same as those illustrated in Fig. 13. Electroless nickel-phosphorus (Ni-P) plating as an important molding material for manufacturing plastic and glass optical components was selected as the workpiece material. The material removal mechanisms under the LV and $\mathrm{CV}$ modes were studied by analyzing the relationship between the textural features and the cutting tip geometries. Figure 14 shows typical textured surfaces under the LV and CV modes, respectively.

Although different textured surfaces have been successfully fabricated by using these electroplated one-point diamond tools, the geometries of the diamond cutting edges are not pre-designed. Thus, the structures cannot be tailored to meet the requirements of practical applications. Many studies have investigated the effect of diamond cutting edge's geometry on the machining performance [23]. However, for conventional cutting processes, the study on the different geometries of diamond cutting edges has mainly focused on the micro-shape of the cutting corner, to reduce tool wear, workpiece surface roughness, and subsurface damage, without considering surface texturing. The tool kinematics of the RUT process is fundamentally different from that of the conventional cutting process. The material removal mechanism is completely different because of the high-frequency periodic change of cutting locus. Therefore, new geometrically defined diamond tools were designed and manufactured to fabricate geometrically defined textures on the basis of the obtained knowledge on material removal mechanisms of RUT. Figure 15 shows two typical geometrically defined diamond tools for RUT processes under the LV and CV modes, respectively.

To analyze the surface generation mechanisms and predict the 3D textural features of textured surfaces, a surface generation model was established for RUT processes under the $\mathrm{LV}$ and $\mathrm{CV}$ modes. Obtaining a simulated textured surface is an effective way of predicting the topography of machined surfaces; however, this strategy does not interpret the tool-workpiece interaction. A better understanding of the tool-workpiece interaction during the material removal process is greatly helpful in designing appropriate tools and obtaining good surface quality. Therefore, we utilized a 3D-CAD software to 
(a)

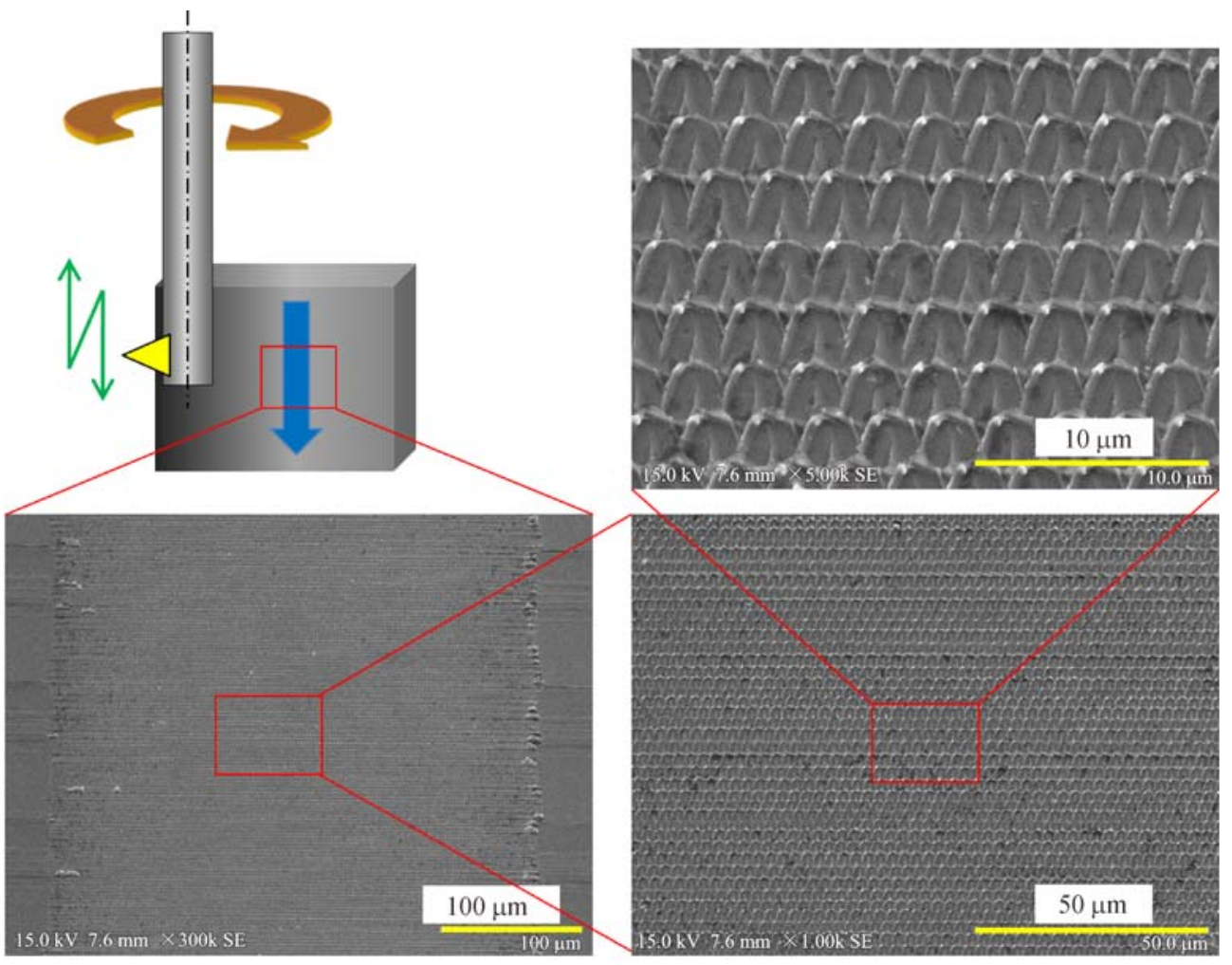

(b)
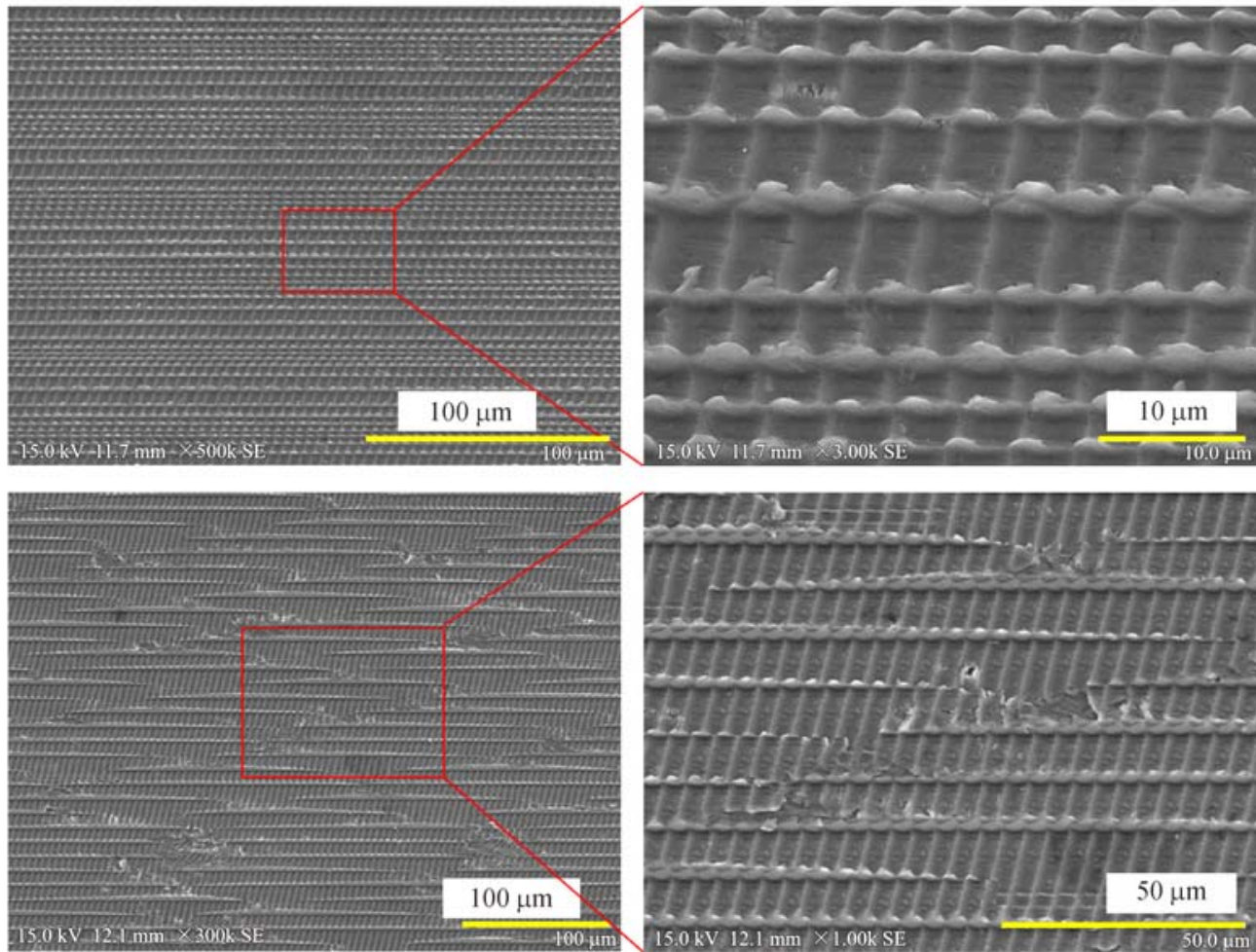

Fig. 14 Typical textured surfaces fabricated by using RUT method under the (a) LV and (b) CV modes using electroplated single-point diamond tools 
(a)

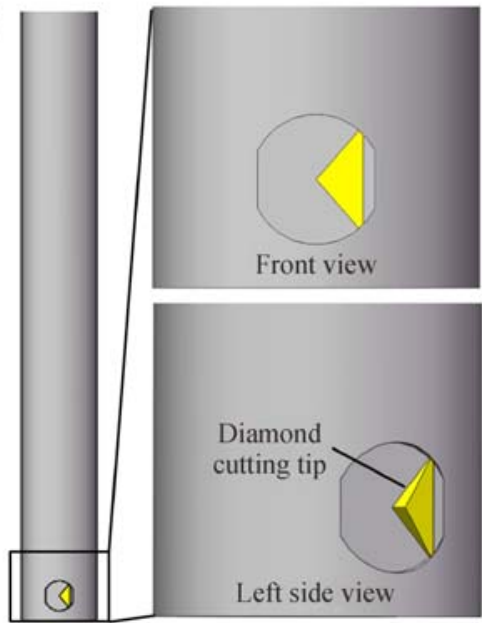

(b)

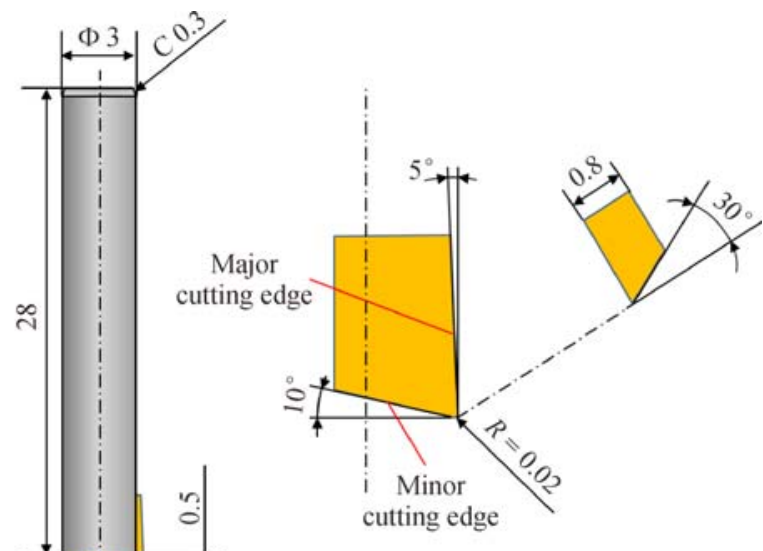

Geometrical shape of the cutting tip

Fig. 15 Two types of geometrically defined diamond tools for the RUT processes under the (a) LV and (b) CV vibration modes. Reprinted from Refs. [11,12] with permission from Springer

(a)
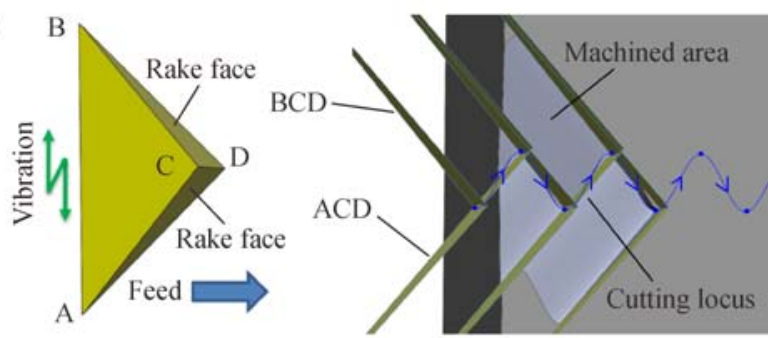

(b)

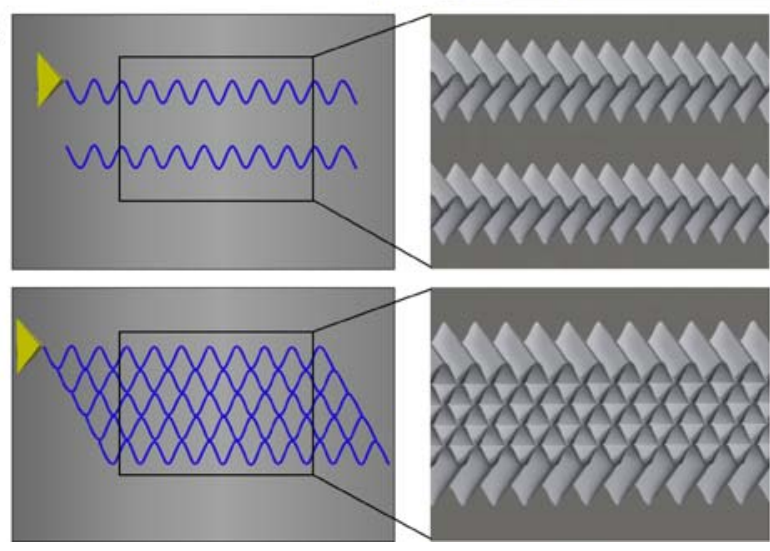

Fig. 16 (a) Discrete steps of material removal processes in RUT and (b) simulated textured surfaces. Reprinted from Ref. [12] with permission from Springer

simulate the surface fabrication process by visualizing every step of tool-work interaction; one example is shown in Fig. 16. Discrete structure fabrication can help designers understand the material removal mechanisms. Figure 17 shows two typical textured surfaces fabricated using the above two geometrically defined tools. The features of the textured surfaces can be predicted by the proposed simulation method, and hierarchical textured surfaces can be generated using the RUT technology.

\subsection{Applications of textured surfaces}

Micro/nano-textured surfaces have been proven useful in many fields because of their functional performance with highly increased surface areas and their novel physical or chemical properties [6]. Nonetheless, the practical application of a specific texturing method is determined by the machinable materials and structures. The RUT method using diamond tools with few limitations at machinable materials can potentially provide solutions in many fields. When using diamond grinding wheels, the RUT method can be used to fabricate structures on the surfaces of hardto-machine materials, such as zirconia ceramics, which is a promising excellent dental restoration material [8]. The textured surface can improve the osseointegration of zirconia dental implants, wherein the regularity of textural patterns is not strict. When using designed single-point diamond tools, the RUT method can be used to generate tailored structures. Thus, the textural patterns can be modulated to obtain specific functions, such as directional textured patterns for inducing directional wetting. Hierarchical micro/nano-structures can highly increase surface areas and consequently induce better hydrophilicity of wettable surfaces. Figure 18 shows a directional wettable textured surface fabricated by using the RUT method under LV mode [12]. The theoretical study of the applications of these textured surfaces should be given more attention, particularly the principles for guiding the design of geometrical features to achieve functional performances.

\section{Problems and challenges}

Although various structures at the micrometer or even nanometer scale have been successfully fabricated using 
(a)

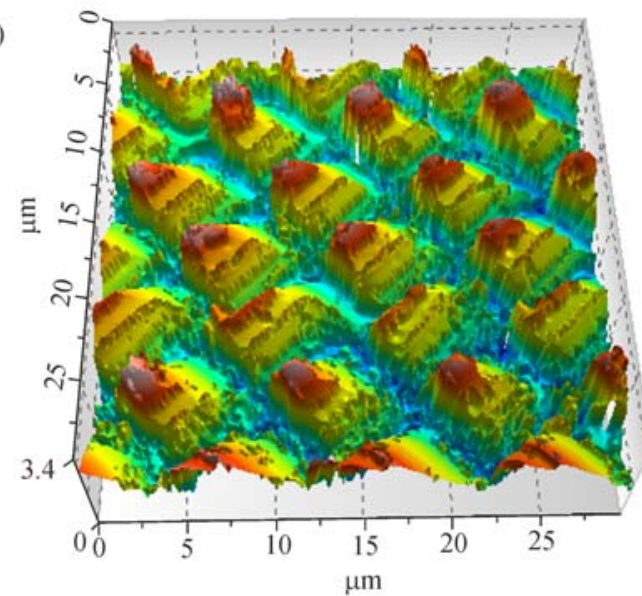

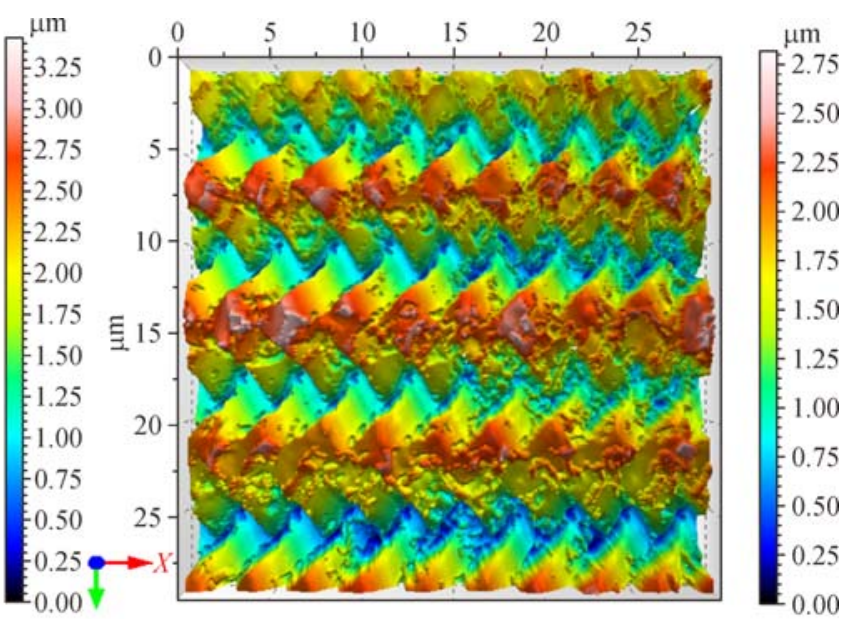

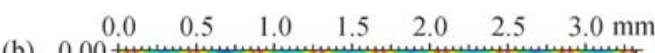
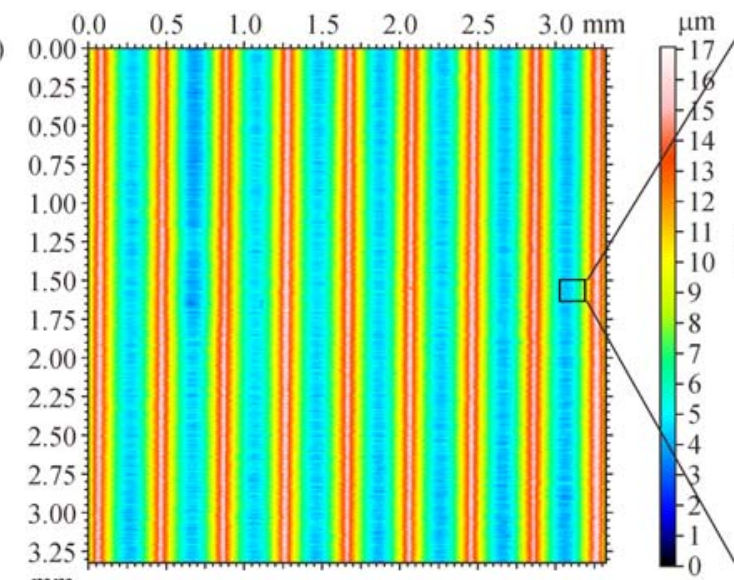

$\mathrm{mm}$
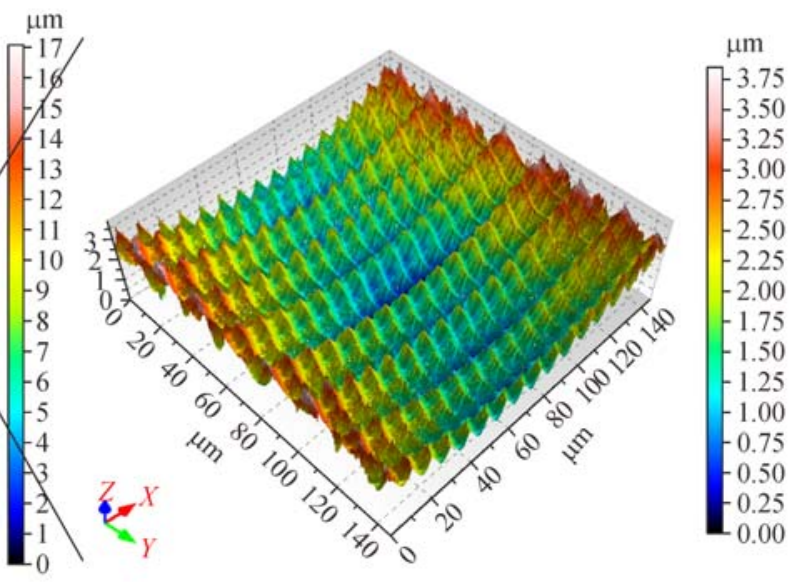

Fig. 17 Typical textured surfaces fabricated by using the RUT method with geometrically defined diamond tools under (a) LV and (b) CV vibration modes. Reprinted from Refs. [11,12] with permission from Springer
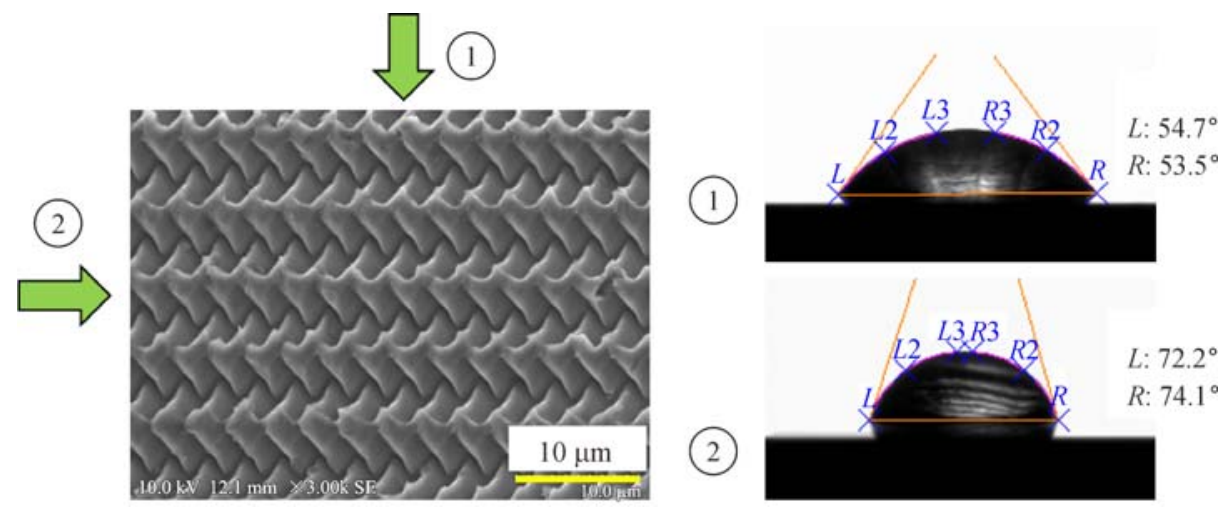

Fig. 18 A textured surfaces and it representative water contact angles, possessing directional wetting properties. Reprinted from Refs. [12] with permission from Springer

the RUT method, it should be noted that its freedom to fabricate structures required in industries is still limited. Integrating a rotary ultrasonic spindle to a machine tool with more degrees of freedom is a possible way of addressing this problem. Many studies have also reported on fabricating more complex structures with the use of a low/fast-servo system by adding more freedoms to a machine tool, and these works have successfully fabricated several hybrid or hierarchical structures [24]. Another strategy is to design diamond tools with more appropriate 
geometries by considering the material removal mechanisms to achieve more machinable structures. Another feasible approach is to further improve the rotary ultrasonic spindle by making it capable of modulating the vibration frequency and the amplitude with more freedom.

Besides, no detailed study is available on the tool wear of ultrasonic-assisted texturing methods. The high-frequency tool-work contact should result in notable tool wear, which must be overcome prior to its industrial application. We proposed to control the depth-of-cut at a constant small value with a designed advanced cutting edge to minimize tool wear [10]. However, this restricts the freedom of tool geometries, thereby limiting the machinable structures. Another way to decrease tool wear is to use tools with better wear resistance. We have attempted to utilize a nano-polycrystalline diamond tool [25] in the RUT processes, and the preliminary results showed that tool wear was much slower than that observed on conventional single crystal diamond tools.

Another problem that should be addressed is that the practical applications of these structures have not been extensively discussed. The principles for modulating the geometrical features of structures to obtain the required functions should be studied with deeper knowledge, which are also scientifically important.

\section{Summary and outlook}

To sum, the proposed ultrasonic-assisted texturing methods can provide designers with more freedom to create various micro/nano-structures for functional performances. These methods are classified into two categories according to the role of the ultrasonic vibration. In addition, this paper introduces a rotary ultrasonic texturing method that modulates the cutting locus at each vibration circle for the fabrication of structures.

The RUT method using a rotary ultrasonic spindle has greater feasibility than other methods, because it can fabricate structures not only on flat surfaces but also on curved surfaces. The geometries of diamond tools can be designed to generate tailored structures, particularly hybrid textured surfaces, to meet requirements of functional performance. The geometrical features of textural patterns can be calculated and simulated.

The RUT method also has a number of limitations. Integrating the rotary ultrasonic spindle into a machine tool of more degrees of freedom can enable the RUT method with more machinable structures. Using nano-polycrystalline diamond tools can address the tool wear problem.

In future works, a more robust and flexible ultrasonicassisted texturing method should be further developed based on the rotary ultrasonic spindle. Moreover, the scientific principle for designing textural patterns for functional performance should be given more attention.
Acknowledgements The work was supported by the Grant-in-Aid for Young Scientists (B) (Grant No. 16K17990) from the Japan Society for the Promotion of Science.

Open Access This article is distributed under the terms of the Creative Commons Attribution 4.0 International License (http://creativecommons.org/ licenses/by/4.0/), which permits unrestricted use, distribution, and reproduction in any medium, provided you give appropriate credit to the original author(s) and the source, provide a link to the Creative Commons license, and indicate if changes were made.

\section{References}

1. Evans C J, Bryan J B. "Structured", "textured" or "engineered" surfaces. CIRP Annals-Manufacturing Technology, 1999, 48(2): 541-556

2. Bauer S, Schmuki P, von der Mark K, et al. Engineering biocompatible implant surfaces: Part I: Materials and surfaces. Progress in Materials Science, 2013, 58(3): 261-326

3. Chung K K, Schumacher J F, Sampson E M, et al. Impact of engineered surface microtopography on biofilm formation of Staphylococcus aureus. Biointerphases, 2007, 2(2): 89-94

4. Tsipenyuk A, Varenberg M. Use of biomimetic hexagonal surface texture in friction against lubricated skin. Journal of the Royal Society Interface, 2014, 11(94): 20140113

5. Bhushan B. Biomimetics: Lessons from nature-An overview. Philosophical Transactions. Series A, Mathematical, Physical and Engineering Science, 2009, 367(1893): 1445-1486

6. Bruzzone A A G, Costa H L, Lonardo P M, et al. Advances in engineered surfaces for functional performance. CIRP AnnalsManufacturing Technology, 2008, 57(2): 750-769

7. Brinksmeier E, Gläbe R, Schönemann L. Review on diamondmachining processes for the generation of functional surface structures. CIRP Journal of Manufacturing Science and Technology, 2012, 5(1): 1-7

8. Xu S, Nishikawa C, Shimada K, et al. Surface textures fabrication on zirconia ceramics by 3D ultrasonic vibration assisted slant feed grinding. Advanced Materials Research, 2013, 797: 326-331

9. Xu S, Shimada K, Mizutani M, et al. Surface texturing and wettability evaluation of zirconia ceramics. In: Proceedings of International Conference on Leading Edge Manufacturing in 21st Century: LEM21. 2013, 7: 208-213

10. Xu S, Shimada K, Mizutani M, et al. Fabrication of hybrid micro/ nano-textured surfaces using rotary ultrasonic machining with onepoint diamond tool. International Journal of Machine Tools and Manufacture, 2014, 86: 12-17

11. Xu S, Shimada K, Mizutani M, et al. Development of a novel 2D rotary ultrasonic texturing technique for fabricating tailored structures. International Journal of Advanced Manufacturing Technology, 2016 (in press)

12. Xu S, Shimada K, Mizutani M, et al. Analysis of machinable structures and their wettability of rotary ultrasonic texturing method. Chinese Journal of Mechanical Engineering, 2016, 29(6): 11871192

13. Denkena B, Kästner J, Wang B. Advanced microstructures and its production through cutting and grinding. CIRP Annals-Manufac- 
turing Technology, 2010, 59(1): 67-72

14. Guo P, Ehmann K F. Development of a tertiary motion generator for elliptical vibration texturing. Precision Engineering, 2013, 37(2): 364-371

15. Guo P, Ehmann K F. An analysis of the surface generation mechanics of the elliptical vibration texturing process. International Journal of Machine Tools and Manufacture, 2013, 64: 85-95

16. Ahmed S A, Hoon Ko J, Subbiah S, et al. Microtexture generation using controlled chatter machining in ultraprecision diamond turning. Journal of Micro and Nano-Manufacturing, 2015, 3(2): 021002

17. Brehl D E, Dow T A. Review of vibration-assisted machining. Precision Engineering, 2008, 32(3): 153-172

18. Suzuki N, Yokoi H, Shamoto E. Micro/nano sculpturing of hardened steel by controlling vibration amplitude in elliptical vibration cutting. Precision Engineering, 2011, 35(1): 44-50

19. Brehl D E, Dow T A. 3-D microstructure creation using elliptical vibration-assisted machining. In: Proceedings of ASPE Spring
Topical Meeting on Vibration Assisted Machining Technology. Raleigh, 2007, 21-26

20. Aurich J C, Egnmann J, Schueler G M, et al. Micro grinding tool for manufacture of complex structures in brittle materials. CIRP Annals -Manufacturing Technology, 2009, 58(1): 311-314

21. Sugai T. Study on ultrasonic vibration cutting. Dissertation for the Master's Degree. Sendai: Tohoku University, 2014

22. Shimada K. Study on Vibration Grinding. Dissertation for the Doctoral Degree. Sendai: Tohoku University, 2012

23. Denkena B, Biermann D. Cutting edge geometries. CIRP AnnalsManufacturing Technology, 2014, 63(2): 631-653

24. Zhu Z, To S, Zhang S. Theoretical and experimental investigation on the novel end-fly-cutting-servo diamond machining of hierarchical micro-nanostructures. International Journal of Machine Tools and Manufacture, 2015, 94: 15-25

25. Harano K, Satoh T, Sumiya H. Cutting performance of nanopolycrystalline diamond. Diamond and Related Materials, 2012, 24 : $78-82$ 